\title{
Coloration determination of spectral darkening occurring on a broadband Earth observing radiometer: Application to Clouds and the Earth's Radiant Energy System (CERES)
}

\author{
Grant Matthews*a, Kory Priestley ${ }^{b}$, Norman G. Loeb ${ }^{b}$, Konstantin Loukachine ${ }^{c}$, Susan \\ Thomas $^{c}$, Dale Walikainen ${ }^{c}$, Bruce A. Wielicki ${ }^{b}$ \\ ${ }^{a}$ Analytical Services and Materials (AS\&M); \\ ${ }^{b}$ Atmospheric Sciences Division, NASA Langley Research Center; \\ ${ }^{c}$ Science Applications International Corporation (SAIC)
}

\begin{abstract}
It is estimated that in order to best detect real changes in the Earth's climate system, space based instrumentation measuring the Earth Radiation Budget (ERB) must remain calibrated with a stability of $0.3 \%$ per decade. Such stability is beyond the specified accuracy of existing ERB programs such as the Clouds and the Earth's Radiant Energy System (CERES, using three broadband radiometric scanning channels: the shortwave $0.3-5 \mu m$, total $0.3->100 \mu \mathrm{m}$, and window $8-12 \mu \mathrm{m})$. It has been shown that when in low earth orbit, optical response to blue/UV radiance can be reduced significantly due to UV hardened contaminants deposited on the surface of the optics. Since typical onboard calibration lamps do not emit sufficient energy in the blue/UV region, this darkening is not directly measurable using standard internal calibration techniques. This paper describes a study using a model of contaminant deposition and darkening, in conjunction with in-flight vicarious calibration techniques, to derive the spectral shape of darkening to which a broadband instrument is subjected. Ultimately the model uses the reflectivity of Deep Convective Clouds as a stability metric. The results of the model when applied to the CERES instruments on board the EOS Terra satellite are shown. Given comprehensive validation of the model, these results will allow the CERES spectral responses to be updated accordingly prior to any forthcoming data release in an attempt to reach the optimum stability target that the climate community requires.
\end{abstract}

Keywords: CERES, climate measurement stability, spectral darkening, optical contamination, atomic oxygen, MODIS, deep convective cloud albedo

\section{INTRODUCTION}

With still considerable uncertainty surrounding the future of climate change, it has recently been determined ${ }^{1}$ that in order to detect possible trends in climate variables, space based measurements of the Earth radiation Budget (ERB) must maintain a calibration stability of $0.3 \%$ per decade. ERB parameters are the scattered short wave (SW $0.3-5 \mu m$ ) and emitted thermal long wave (LW $5->100 \mu m$ ) radiative flux from the Earth. Such global ERB parameter measurements are currently made by the Clouds and the Earth's Radiant Energy System $\left(\mathrm{CERES}^{2}\right)$, a broadband satellite radiometer program intended to resolve many of the remaining uncertainties surrounding the role of clouds in ERB and future climate change. However the calibration stability now required by the climate modeling community is beyond the design specifications of any existing ERB mission, including CERES. Achieving such stability becomes most challenging given that optics used in missions such as CERES are subject to significant optical darkening, ${ }^{3}$ reducing the response to SW radiance in a manner not directly detectable using standard calibration techniques. The extent of the darkening on each CERES instrument has been estimated ${ }^{3}$ and so called 'rev1' modification factors have been released to the climate community (via the CERES data quality summaries ${ }^{4}$ ) to make adjustments to existing CERES data. For the most part, these adjustments have proved most successful in removing spurious trends ${ }^{5,6}$ in CERES data caused by calibration insensitivity to spectral darkening. However, this study details the development of a new calibration methodology that combines vicarious calibration with a physical model of contamination induced optical degradation. The intention is to assign spectral coloration to the changes found in the response of a broadband radiometer to Earth radiance.

*fn.g.matthews@larc.nasa.gov, voice: 1757827 4894, 1 Enterprise Parkway, Suite 300, Hampton, Virginia 23666 

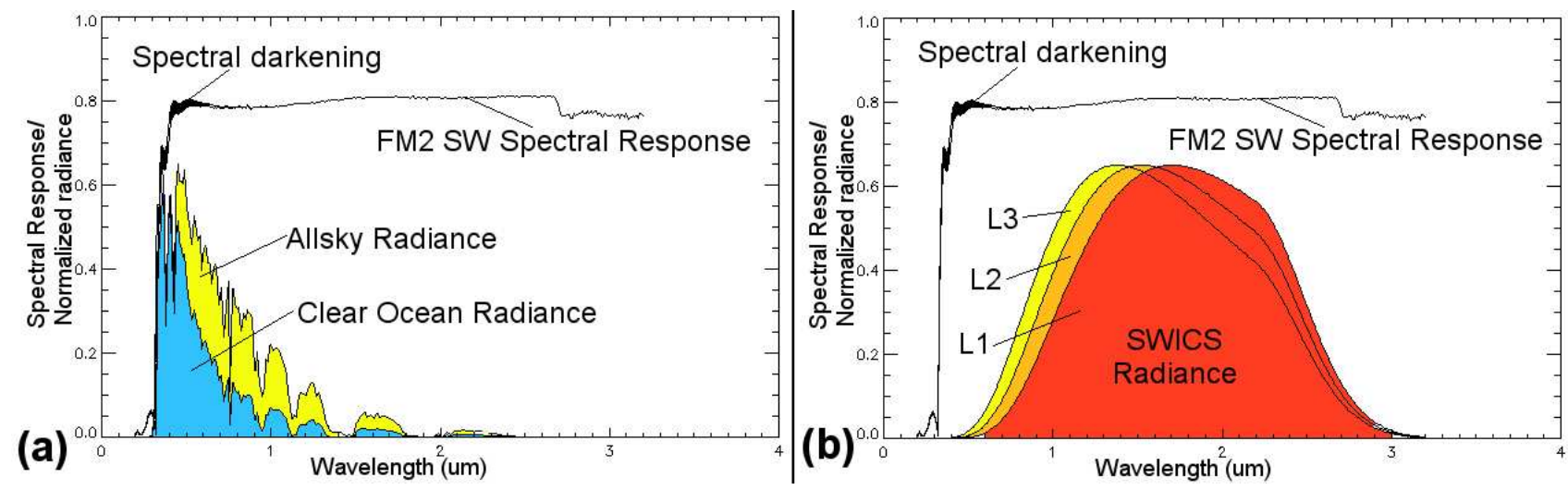

Figure 1. (a) Comparison of normalized clear ocean and all-sky spectra with a CERES spectral response subject to spectral darkening. (b) Comparison of the 3 levels of normalized tungsten lamp output with a CERES spectral response subject to spectral darkening.

\section{SPECTRAL DARKENING EFFECT ON CERES BROAD-BAND OPTICS}

Four CERES instruments are currently operational, Flight Model 1 (FM1) and 2 (FM2) are onboard the Earth Observing System (EOS) platform 'Terra', launched in December 1999. FM3 and FM4 operate onboard the EOS 'Aqua' platform launched in May 2002. Both Terra and Aqua are in high inclination near polar, sun synchronous orbits. Terra crosses the equator at 10:30 am local time in the descending portion of the orbit while Aqua ascends across the equator at 1:30 pm local time. At all times one of the CERES units on each platform operates in crosstrack mode with the scan plane perpendicular to the direction of travel. Until recently the other unit was placed in rotating azimuth plane (RAPS) mode, where the scan plane was rotated continuously to allow the instrument to view specific targets with varying geometry (for purposes of developing angular dependency models ${ }^{7}$ ). The broadband scanning CERES radiometer instruments have 3 radiometric channels that are the SW $(0.3-5 \mu m)$, window (WN $8-12 \mu \mathrm{m})$ and total $(0.3->100 \mu \mathrm{m})$. The SW channel uses a spectral filter (quartz fused silica) for blocking longwave radiance. The daytime LW measurement is obtained by subtracting the SW measurement from that made by the total channel which has no filter. Each channel uses a silver coated Cassegrain mirror telescope to focus radiance onto a painted black thermistor bolometer detector. Onboard calibration sources are used for every channel, with concentric groove blackbodies for the window and total channels and a stable tungsten lamp for the SW channel. In the original calibration principal on which CERES was designed, the lamps were to be used to determine shifts in the SW channel gain from ground calibration when first in orbit. However, a tungsten lamps emissions are concentrated primarily in the near infra-red (Fig. 1(b)). Hence when broadband optics such as these are subjected to spectral darkening, ${ }^{3}$ a significant drop in response to earth scene radiance will occur (Fig. 1(a)) while negligible change will be seen ${ }^{8}$ in the response to the CERES SW internal calibration source (SWICS, Fig. 1(b)). The CERES instruments were therefore equipped with Mirror Attenuator Mosaic (MAM) diffusers. These direct solar radiance on the SW and total channel optics to allow monitoring of changes in response to radiance at wavelengths in the visible and UV spectral regions (see Fig. 2(a)). Hence the intention was to use fortnightly solar calibrations through the MAMs to determine drifts in the SW response of the CERES optics. Unfortunately the fabrication of the MAM diffusers did not meet specifications and soon after launch it became clear that their reflectivity was itself changing by an order of magnitude greater than the stability required in SW measurements. This deprived the CERES instruments of their primary SW stability metric. Till now the extent of the spectral darkening could only be determined using comparisons of simultaneous nadir measurements between the degrading RAPS instrument on Terra or Aqua and that locked in cross track (i.e. the cross-track unit was used as a transfer radiometer since it is not subject to ram exposure, believed to be a primary cause of optical contamination). Fig. 2(c) shows how the instrument operating in RAPS mode, that is exposed to the ram direction, always drops in response relative to the instrument held in cross-track. Experience gained form the Long Duration Exposure Facility mission (LDEF, ${ }^{9}$ that returned optical samples from orbit via the shuttle, see Fig. 3(a)) suggests that exposure of optics to the ram direction allows highly reactive atomic oxygen to mobilize contaminant molecules from coatings such as paints used on telescope baffles. 


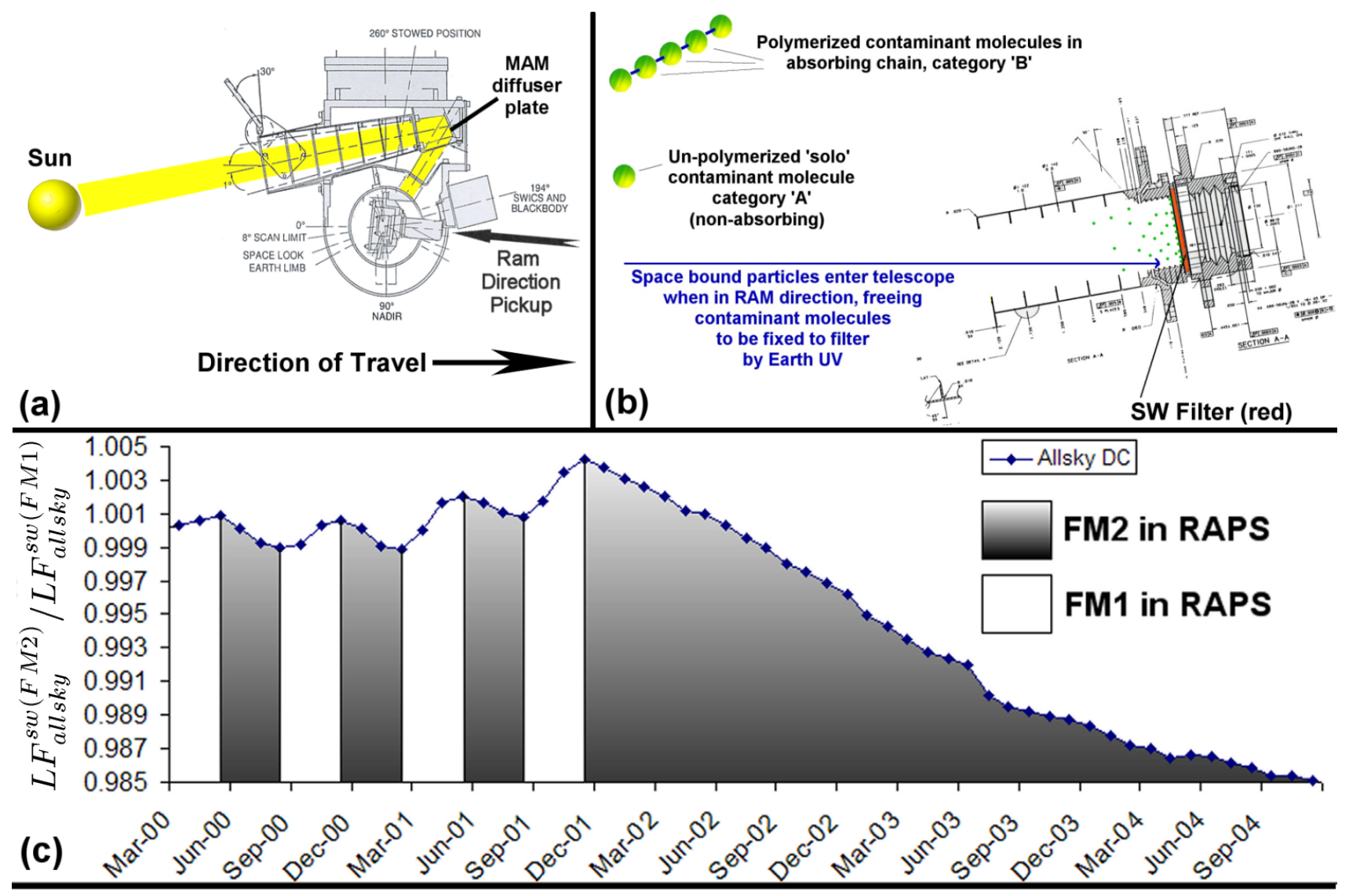

Figure 2. (a) Geometry of CERES solar calibrations using MAM and exposure of telescope to ram direction when aligned in 'along-track'. (b) Theoretical cause of contaminant arrival while telescope points in ram direction. (c) Direct compare ratio between simultaneous filtered SW all-sky FM2 and FM1 nadir measurements.

These contaminant molecules then can be fixed to an optical surface when exposed to UV photons. Continued exposure to UV radiance will cause any remaining solo contaminant molecules (category ' $A$ ' which cause little to no absorption) to polymerize into long highly absorbing chains, at which point they are considered category ' $B$ ' (see Fig. 2(b)).

For the Edition 2 data release, the gain of the CERES SW channels was updated based on the change in signal from the onboard SWICS lamps. The rev1 adjustment then assumed that the cross track instrument underwent no darkening and so could be used to measure the degradation of the other RAPS instrument. Comparisons ${ }^{6}$ with the highly stable SeaWiFS ${ }^{10}$ instrument showed the rev1 adjustment to be highly successful for CERES measurements from the Terra platform. However, even after rev1 adjustments were applied the CERES data from the Aqua satellite, they were seen to drop compared to measurements from their counterparts on Terra. This suggests that the rev1 adjustments were not so successful in maintaining the stability of the Aqua CERES instruments. This can be for one or both of two reasons. It is possible that the lamps on the Aqua instruments got brighter in time (as seen on the PFM TRMM instrument), causing the gain to be incorrectly raised in the Edition 2 release. It is also possible that the cross-track instrument undergoes significant darkening without ram exposure, perhaps due to continued earth scattered UV induced polymerization of contaminant molecules (i.e. conversion of remaining category $A$ molecules to category $B$, see Fig. 2(b)). It is therefore the intention of this study to use a physical model of contaminant mobilization and polymerization, combined with the stability metric of deep convective cloud albedo, to derive changes to the instrument gain and spectral response shape. This model will then be tuned by matching all-sky and clear ocean direct compare data. This should ensure inversion of CERES data that will provide stability of the order required by the climate community. 

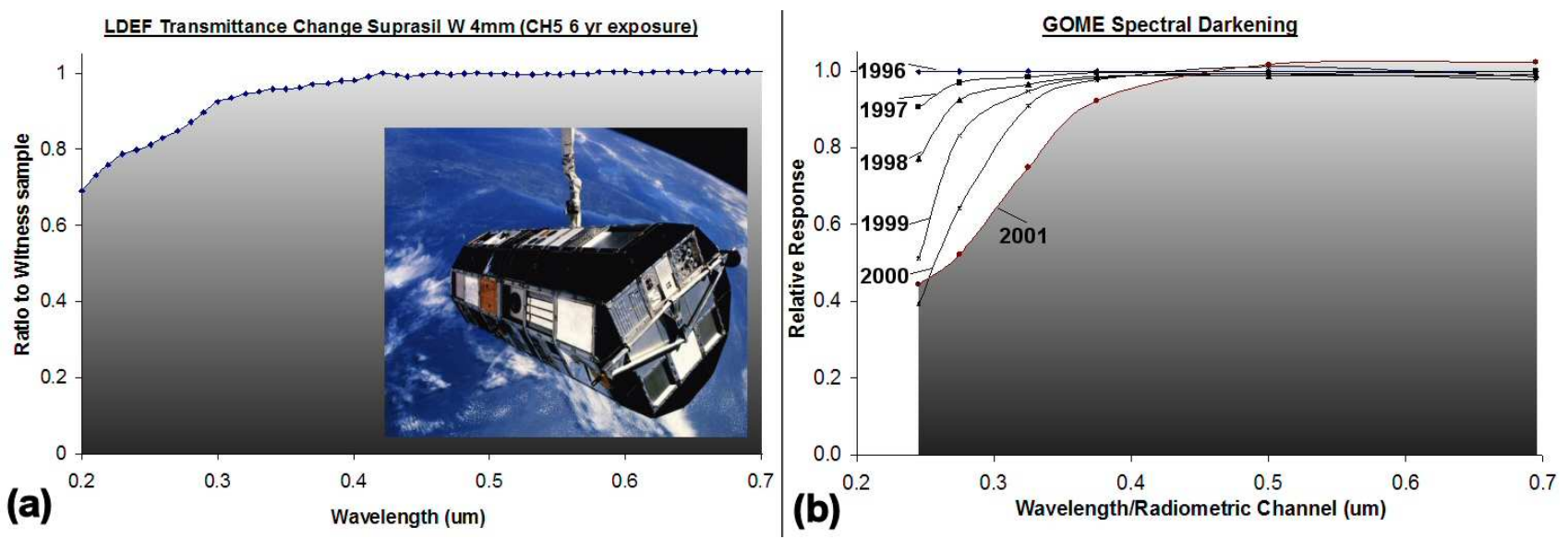

Figure 3. (a) Change in spectral transmission of fused silica (Suprasil) dome on LDEF mission. (b) Change in response of narrowband spectral channels on GOME mission throughout lifetime.

\section{METRIC FOR SW STABILITY: FILTERED DEEP CONVECTIVE CLOUD ALBEDO}

With CERES MAM reflectivity seen to change significantly and the onboard lamp output containing insignificant UV energy, a replacement stability metric is required to fill the place intended for the solar MAM calibrations. It has been shown that regular views of the moon can be used to maintain visible radiometer stability. ${ }^{11}$ However the fully illuminated lunar disk fills less than $10 \%$ of the CERES field of view and lunar brightness is known to vary significantly at the different phase angles when CERES lunar scans have occurred. Hence it is not currently possible to use these data as a stability metric with the precision required. Since CERES has not the dynamic range to stare straight at the Sun, what is required is a solar reflecting target that can fill the entire CERES field of view and maintains a consistent albedo on a decadal timescale.

The most consistent reflector available to CERES has been deemed to be Deep Convective Clouds (DCC). These are large, very optically thick clouds that form in tropical regions. The tops of these clouds reside at very high altitudes (above $10 \mathrm{~km} / 300 \mathrm{mb}$ ) and consist entirely of ice particles due to the extreme low temperatures. With reflective tops at such high altitudes, they are above the majority of atmospheric absorption, and are the closest significant atmospheric reflector to space (except for ozone). Therefore in this study, cloud properties contained in the CERES single scanner footprint (SSF) data product have been used to identify when the CERES instrument is viewing DCC. These filtered SW measurements are then sub-setted for use in monitoring stability (SSF cloud properties are derived using measurements from the MODerate resolution Imaging Spectrometer, MODIS, ${ }^{12}$ operating on the same platform as CERES). It is important to distinguish between the un-filtered SW radiance product ' $L_{i}^{s w}$ ' and the CERES measurement of filtered SW radiance ' $L F_{i}^{s w}$ ' made when viewing scene ' $i$ ':

$$
\begin{aligned}
L_{i}^{s w} & =\int_{0}^{\infty} b_{i}(\lambda) I^{s}(\lambda) d \lambda \\
L F_{i}^{s w} & =g_{s w} \int_{0}^{\infty} r_{s w}(\lambda) b_{i}(\lambda) I^{s}(\lambda) d \lambda \\
f_{i} & =\frac{\int_{0}^{\infty} r_{s w}(\lambda) b_{i}(\lambda) I^{s}(\lambda) d \lambda}{\int_{0}^{\infty} b_{i}(\lambda) I^{s}(\lambda) d \lambda} \\
L_{i}^{s w} & =\frac{L F_{i}^{s w}}{g_{s w} \times f_{i}}
\end{aligned}
$$

$I^{s}(\lambda)$ is the solar radiance incident at the Earth and $b_{i}(\lambda)$ is the spectral albedo of scene $i$. The filtered radiance measurement represented in Eqn. 2 is found from detector volts divided by ground measured gain (i.e. if there is no gain change $g_{s w}=1$ ). The job of in-flight calibration for CERES involves determining changes in the 

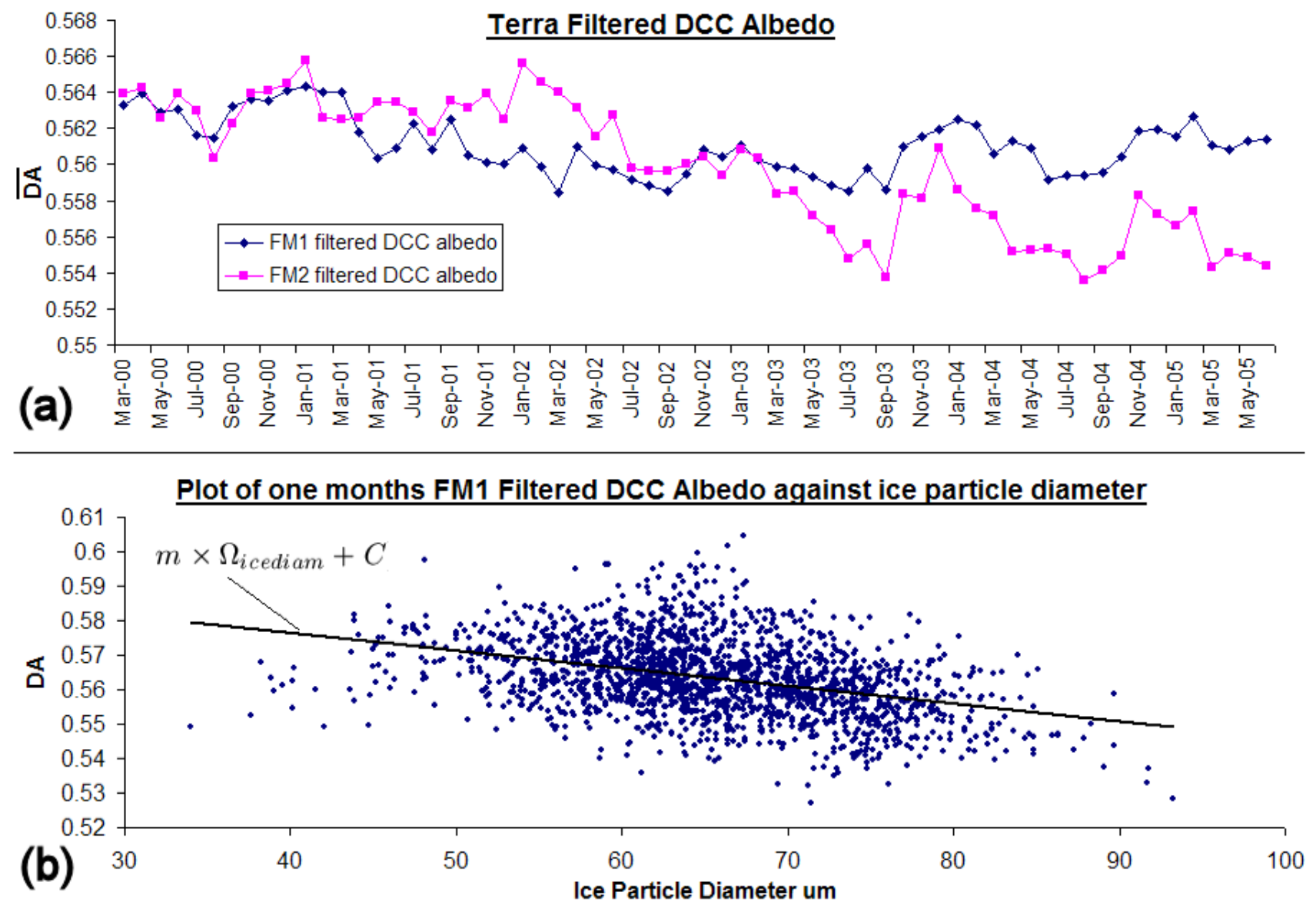

Figure 4. (a) Monthly averaged Deep Convective cloud filtered albedo $\overline{D A}$ (Eqn. 5) as measured by the Terra CERES instruments. MODIS cloud properties and radiances are then used to assign a DCC spectrum to each data point based on the ModTran database. (b) Regressed plot of one months FM1 filtered DCC albedo against ice particle diameter.

bolometer gain $g_{s w}$ and spectral response $r_{s w}(\lambda)$ from their ground measured values. This allows recovery of the unfiltered ERB parameter (Eqn. 1) from Eqn. 4 using the gain and theoretical filtering factor $f_{i}$ (Eqn. 3 calculated from ModTran spectra $\left.{ }^{13}\right)$.

This work utilizes the filtered radiance measurement from DCC as a diagnostic in finding the changes to $g_{s w}$ and $r_{s w}(\lambda)$ (Eqn. 2). The intention is to ensure that the calibration inversion of Eqn. 4 maintains the stability of the unfiltered product to $0.3 \%$ per decade. MODIS information within the SSF product is used to identify tropical ocean DCC $\left( \pm 30^{\circ}\right.$ latitude), whose cloud tops have a temperature of less than $205 \mathrm{~K}$, optical thickness $\tau>120$ and MODIS imager $0.6 \mu m$ radiance standard deviation of less than $3 \%$ (this assures large and uniform reflectivity). The filtered DCC albedo is then found using Eqn. 5:

$$
D A=\frac{L F_{d c c}^{s w} \times A R\left(\theta_{v z}, \phi_{r a z}, \Phi_{s z}\right) \times D M\left(\Phi_{s z}\right)}{F_{s}} \times\left(\frac{Z}{Z_{0}}\right)^{2} \times\left(\frac{m \times 65 \mu m+C)}{\left.m \times \Omega_{\text {icediam }}+C\right)}\right)
$$

where $\theta_{v z}, \phi_{r a z}$ and $\Phi_{s z}$ are the viewing zenith, relative azimuth and solar zenith angles respectively. The state of the art CERES anisotropic factor $A R\left(\theta_{v z}, \phi_{r a z}, \Phi_{s z}\right)$ is used to convert radiance to flux and $D M\left(\Phi_{s z}\right)$ is the CERES TRMM derived directional model used to adjust reflectivity to that of over head sun $\left(\Phi_{s z}=0\right)$. $Z$ is the Earth-Sun distance at the time of measurement, $Z_{0}$ is one astronomical unit of distance and $F_{s}$ is the solar constant in $W^{-2}$ derived from the RMIB composite. ${ }^{14} \quad \Omega_{\text {icediam }}$ is the DCC ice particle diameter for that footprint and $m$ and $C$ are found by regressing all $D A$ footprints for that month against individual ice particle 

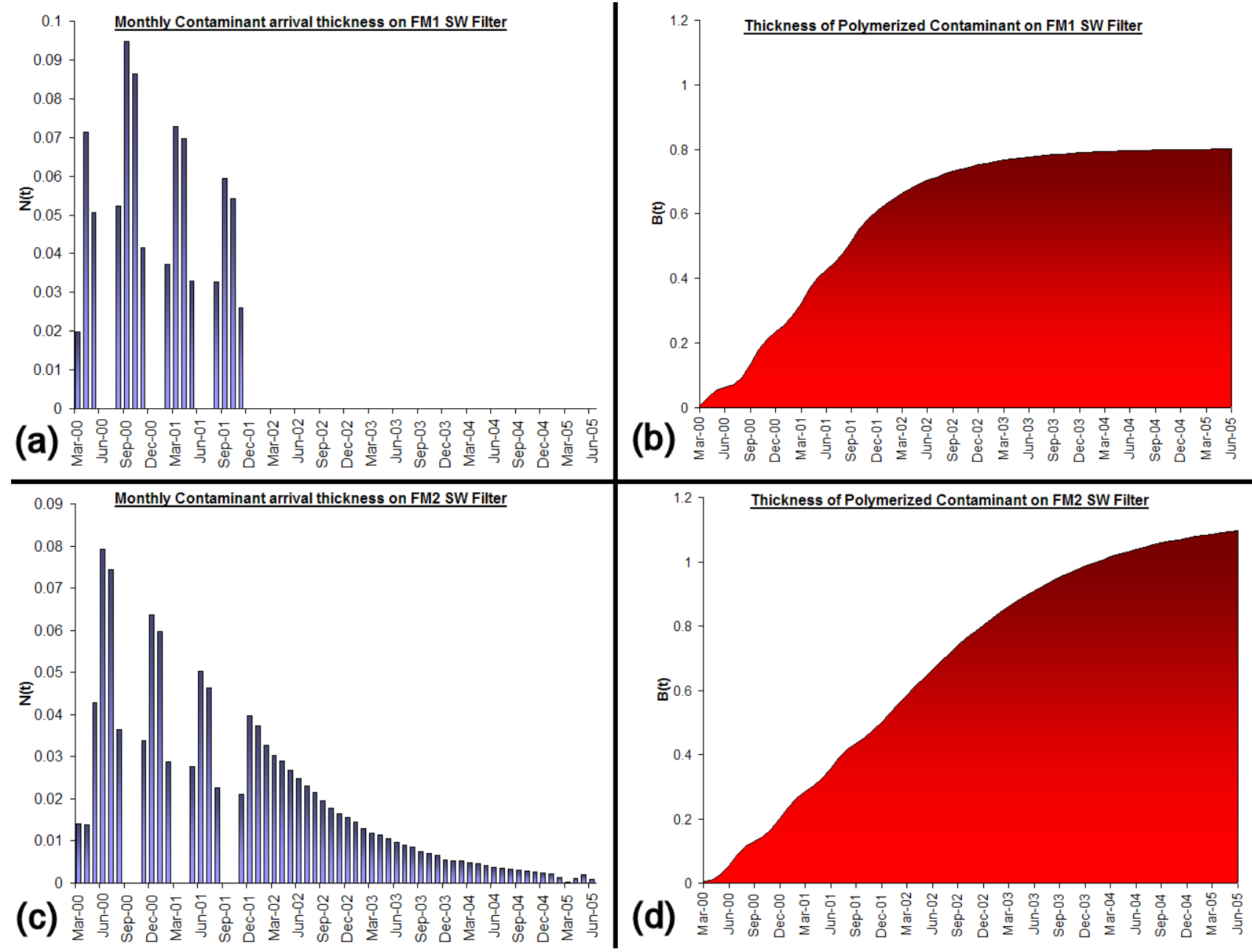

Figure 5. (a) Thickness $N(t)$ of contaminant arriving on FM1 SW filter during each month of mission. (b) Thickness $B(t)$ of polymerized absorbing contaminant on FM1 SW filter. (c) Thickness $N(t)$ of contaminant arriving on FM2 SW filter during each month of mission. (d) Thickness $B(t)$ of polymerized absorbing contaminant on FM2 SW filter.

diameters (Fig. 4(b)). This last term then effectively normalizes the albedo to be that obtained from a DCC with ice particle diameter of $65 \mu \mathrm{m}$ and is acceptable because the ice particle diameter retrieval relies on the MODIS 3.7 $\mu \mathrm{m}$ channel (whose calibration uses an onboard blackbody). Such normalization should prevent any variations in ice particle diameter and hence DCC reflectance from impacting the stability of the CERES SW channel (e.g. in the event of a large volcanic eruption that alters nucleation and DCC ice particle size).

For each instrument, MODIS typically identifies around 5000 DCC footprints per month that meet the required criteria of temperature, uniformity and optical depth. The monthly averages of Eqn. 5 are plotted in Fig. 4(a). Assuming that the solar flux composite $F_{s}$ values are stable and that the albedo of DCCs meeting the criteria are constant, then the trends in Fig. 4(a) are entirely due to changes in the instrument values of $g_{s w}$ and $r_{s w}(\lambda)$. Although there is a definite $0.5 \%$ month to month noise in the result, for an Earth target measurement that has not undergone any de-seasonalization the result is remarkably stable. This then takes the place intended for the MAM solar calibration data, becoming the metric by which CERES stability is maintained. Precisely how stable the albedo of DCC is should be verified by a future study, perhaps utilizing the measurements of new or existing instruments such as SeaWiFS. 


\section{MODEL OF CONTAMINANT TRANSMISSION, DEPOSITION AND POLYMERIZATION}

As shown in Fig. 3, experience from previous missions such as $\mathrm{LDEF}^{9}$ and the narrowband Global Ozone Monitoring Experiment $\left(\mathrm{GOME}^{15}\right)$ suggests that space bound optical contaminant absorption increases with shorter wavelength. The darkening model developed here must therefore represent mathematically how this absorption varies with wavelength. Typically, radiation tends to interact most strongly with targets of size similar to the wavelength of the radiant photons. The LDEF experience suggests space bound contaminants polymerize into lengthy absorbing molecular chains. It is therefore expected that the contaminant absorption $a(\lambda)$ be proportional to the population density of polymerized molecule chains of length $\lambda(P(\lambda)$, Eqn. 6). From statistical mechanics, the population density of a molecule drops off exponentially with its energy level $E$ (i.e. density $\propto e^{-\frac{E}{k T}}$, with $k$ Boltzmann's constant and $T$ the absolute temperature). Assuming that the length of a polymerized chain length is proportional to is energy level $E$, then the contaminant population density and hence its absorption also falls off exponentially with increasing length/wavelength $\lambda($ Eqns. 7, 8)

$$
\begin{aligned}
a(\lambda) & \propto P(\lambda) \\
a(\lambda) & \propto e^{-\alpha \lambda} \\
a(\lambda) & =M \cdot e^{-\alpha \lambda} \\
T r(\lambda) & =1-M \cdot e^{-\alpha \lambda}
\end{aligned}
$$

The transmission $\operatorname{Tr}(\lambda)$ of a 'unit thickness' of polymerized contaminant is found in Eqn. 9 as the fractional absorption subtracted from one ('unit thickness' is a dimensionless parameter related to optical thickness $\tau$, since $\tau$ is found from the natural logarithm of Eqn. 9). This mathematical form agrees very well with the change in transmission seen on both LDEF and GOME (Fig. 3)

Given the Eqn. 9 representation of polymerized contaminant transmission, the model must now simulate how the thickness of contaminant molecule type ' $B$ ' (Fig. 2(b)) increases based on the amount of time exposed to the ram direction and continuous polymerization by Earth scattered UV. The model therefore assumes that contaminant is mobilized to arrive at the optics only when they are exposed to the ram direction. Upon arrival a fraction ' $\beta$ ' of the molecules are already polymerized into absorbing chains. Then every day, a fraction ' $\rho$ ' of the remaining un-polymerized and un-absorbing type ' $A$ ' molecules polymerize with UV exposure to become type ' $B$ ' absorbers. Hence if $N(t)$ is the thickness amount of contaminant mobilized to the optic on a particular day, the rate of change in thickness of absorbing molecule layer $B(t)$ is given by Eqn. 10:

$$
\frac{d B(t)}{d t}=\rho\left[\int_{0}^{t} N(\xi) d \xi-B(t)\right]+\beta \cdot N(t)
$$

An estimate of $N(t)$, or how much contaminant arrives on the optics during a day of ram exposure, must therefore be provided. It is assumed that the contaminant is mobilized from finite a reservoir at a rate that is proportional to the amount remaining. Hence if the total amount of contaminant contained in the reservoir at the start of mission is $Q$, the arrival rate after time $t_{r}$ spent exposed to the ram direction is given by Eqn. 11:

$$
N\left(t_{r}\right)=\psi\left[Q-\int_{0}^{t_{r}} N(\xi) d \xi\right]
$$

Given the time each Terra instrument telescope has spent exposed to the ram direct in each month of the mission, it is therefore possible to estimate how much contaminant arrived in that particular month (Figs. 5(a) \& (c)). As is illustrated in Fig. 5(c) for the FM2 instrument (which operated in RAPs mode continuously from Nov 01 to June 05) this resulted in an exponential fall off of contaminant arriving every month because less and less contaminant remained in the reservoir due to continuous mobilization.

With knowledge of contaminant arrival rate $N(t)$, which acts as a forcing function to Eqn. 10, it is possible to use a $\mathrm{z}$ domain derived recursive filter ${ }^{16}$ and simulate the thickness of polymerized contaminant $B(t)$ on the SW optics for each month of the mission. Such simulations for the Terra instruments are shown in Figs. 5(b) \& (d). These illustrate how after the FM1 instrument was locked in cross-track mode from Nov 01 onwards, arrival 
of contaminant ceased and hence the thickness $B(t)$ then approaches an asymptote as the remaining type ' $A$ ' molecules continue to polymerize into the type ' $B$ ' absorbers. However, on the FM2 instrument the thickness of molecule $B$ absorbers continues to grow due to prolonged exposure to ram direction (Fig. 5(d)). Eventually a fall off occurs towards the end of the period due to the contaminant reservoir becoming depleted and reducing the monthly arrival rate $N(t)$ (Fig. $5(\mathrm{c})$ ).

Since contaminant optical thickness is found as the natural logarithm of Eqn. 9, the SW channel spectral response in month $t$ of the mission can now be derived using:

$$
\begin{aligned}
D(\lambda, t) & =\left[1-M . e^{-\alpha \lambda}\right]^{B(t)} \\
r_{s w}(\lambda, t) & =D(\lambda, t) \times R_{s w}(\lambda)
\end{aligned}
$$

where $R_{s w}(\lambda)$ is the SW channel spectral response as measured in the ground calibration.

\section{TUNING THE DARKENING MODEL TO MATCH VALIDATION DATA}

With the development of a physical model that simulates how the spectral response $r_{s w}(\lambda)$ of the CERES SW channels changes given the time spent operating in various modes, a method of determining model parameters such as contaminant amounts and arrival rates must be found. The tuning of the model will consider two fundamental boundary conditions. Firstly, the models estimate of changes to the spectral response must be accompanied with a gain change that provides a constant DCC unfiltered albedo (i.e. the trend in $\overline{D A}$ matches exactly the model estimate of $g_{s w} \times f_{d c c}$ ). This matching of the long term trends in $\overline{D A}$ ensures that CERES data obtains stability and accuracy of its absolute calibration. However the result of $\overline{D A}$ is far two noisy on short time scales for tuning of the models simulation of RAPS versus cross-track darkening (see Fig. 2(c) and note how the initial 3 monthly oscillation is not discernable in the result of Fig. 4(a)). Hence the model precision will be gained by matching its estimates of direct compare to the second boundary condition of actual measured values for all-sky (Fig. 2(c)) and clear ocean. Use of two scenes of radically different spectra (Fig. 1(a)) ensures accuracy in the model estimate of coloration (i.e. the values of $M$ and $\alpha$ in Eqn. 9). In summary, the model tuning will utilize DCC filtered albedo to obtain absolute stability and accuracy, while obtaining month to month precision by matching the all-sky and clear ocean direct compare.

Such tuning requires one more important piece of information, in that it must be known how the different scenes of DCC, all-sky and clear ocean vary in their spectral content. Neither the Terra nor Aqua platforms have high resolution spectrometer measurements co-located with the CERES footprint, meaning that there are no measurements of the spectrum CERES is viewing to feed into the model. Since MODIS enables determination of the exact IGBP scene type and detailed cloud properties, it should be possible to get an accurate representation of scene spectra from the ModTran database. However the existing SSF information does not include details on atmospheric aerosol content (which can significantly alter albedo $b_{i}(\lambda)$ ). So given all the possible ModTran spectral simulations of the different aerosols for a particular scene $i$, the spectral shape that best matches the relative radiances from the MODIS $0.6,0.8$ and $1.6 \mu \mathrm{m}$ channels is chosen as the most likely scene spectrum.

This study assumes that the unfiltered DCC albedo is constant. That being the case, if the monthly averaged filtered albedo result $\overline{D A}$ from Eqn. 5 were used in place of $L F_{i}^{s w}$ in Eqn. 4, along with the correct gain change $g_{s w}$ and filtering factor $f_{d c c}$ (Eqn.3), the result must also be a constant. Using a re-arrangement of Eqn. 4 this allows determination of the gain change that must accompany the model estimates of $r_{s w}(\lambda, t)$ and $f_{d c c}(t)$ :

$$
\begin{aligned}
\frac{\overline{D A}(t)}{f_{d c c}(t)} & =U D A \times g_{s w}(t) \\
& =U D A\left[1+\Delta g\left(1-e^{t / \tau_{g}}\right)\right]
\end{aligned}
$$

where $U D A$ is the assumed constant of unfiltered DCC albedo (typically a value of 0.725 measured by Terra). The gain of a bolometer is inversely proportional to its thermal mass. ${ }^{16}$ Since this decreases exponentially in the vacuum of space due to out-gassing of air bubbles, the change to a space bound bolometer gain can be modeled by Eqn. 15 (with $\Delta g$ the asymptotic fractional gain change and $\tau_{g}$ the time constant of out gassing from the 


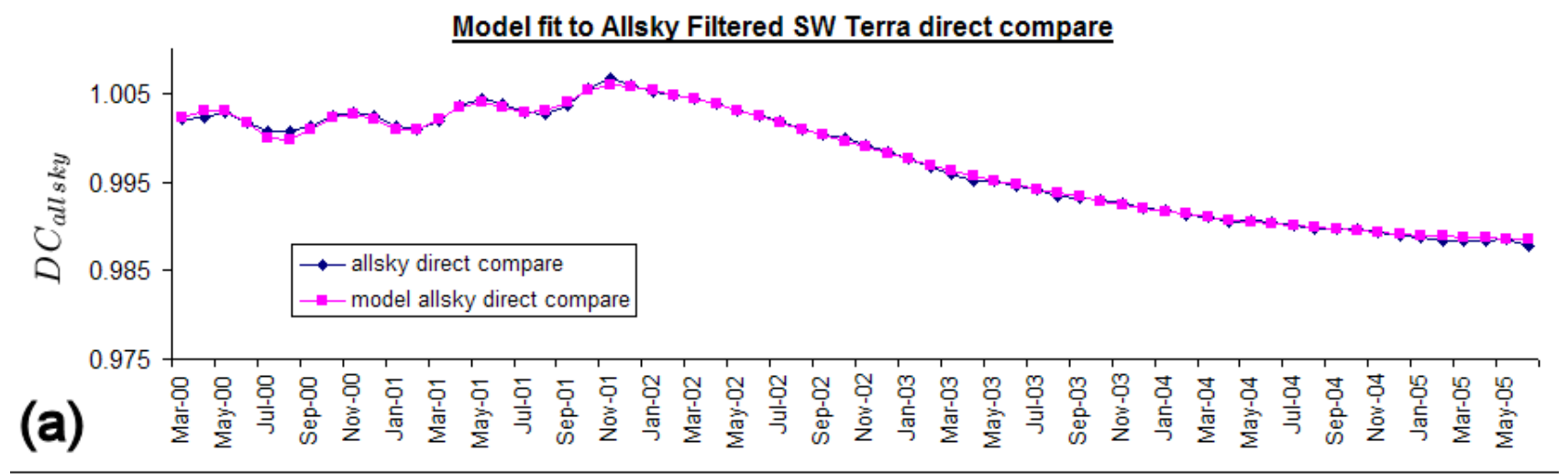

Model fit to Clear Ocean Filtered SW Terra direct compare

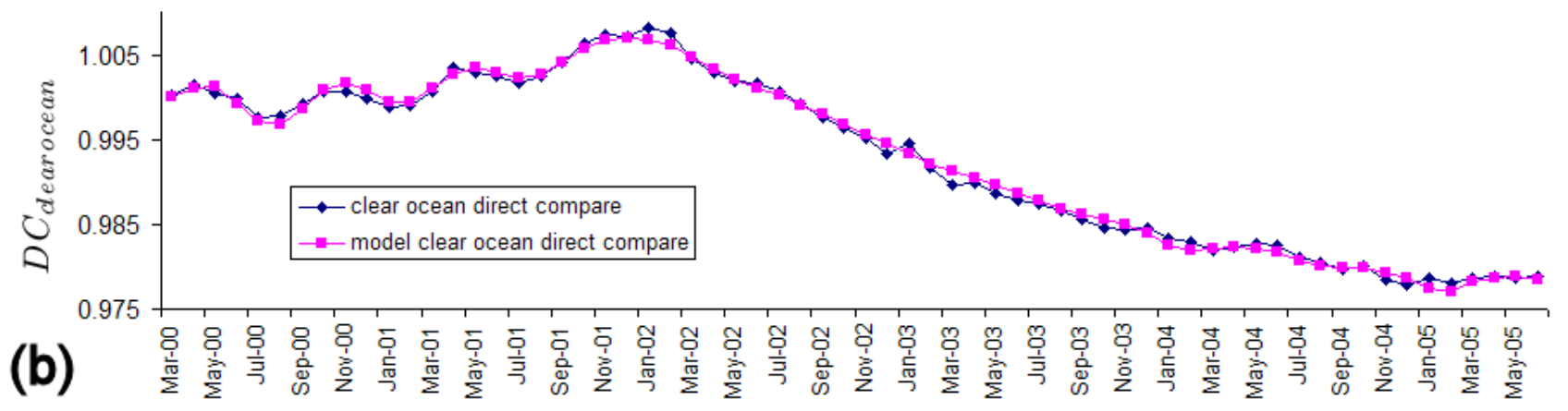

Figure 6. (a) Model fit to all-sky direct compare of nadir footprints. (b) Model fit to clear ocean direct compare of nadir footprints.

bolometer). Hence the change to the SW channel radiometric gain is found using an exponential curve fit to the result of Eqn. 14.

This method of deriving the in-flight gain change ensures that for all model parameters, a gain and spectral response is obtained that will ensure CERES measurements of DCC albedo contain no trends at all. The actual tuning of the model uses a comparison of its estimates of allsky and clear ocean filtered direct compare with the measured values (found as $L F_{i}^{s w(F M 2)} / L F_{i}^{s w(F M 1)}$, the ratio of near simultaneous nadir footprints by the two instruments on the same EOS platform viewing scene $i$ ). This ratio can be simulated using the model estimates of gain and spectral response for the two different instruments thus:

$$
D C_{i}(t)=\frac{g_{s w}^{2}(t) f_{i}^{2}(t)}{g_{s w}^{1}(t) f_{i}^{1}(t)}
$$

Hence the model parameters are iteratively adjusted until Eqn. 16 yields the best possible match to the actual ratio of filtered radiance for the two scenes of all-sky and clear ocean. Fig. 6 shows the result of the tuning, how the model manages to successfully recreate the oscillatory nature of the direct compare prior to Nov 01 when the instruments each spent 3 months at a time exposed to the ram direction. Then after this period, the divergence of FM1 and FM2 is recreated very accurately, with the greater divergence found for clear ocean scenes with a higher proportion of radiant energy in the blue/UV region.

Fig. 7 illustrates the model estimates of changes in instrument response from the start of mission to Jun 05 (instrument response is defined here as the product of gain change and spectral response $g_{s w} \times r_{s w}(\lambda)$ ). This suggests the FM2 SW optics have darkened considerably more than those on FM1 because of greater time exposed to the ram direction. The model has also been successfully used to derive gain and spectral response calibration parameters for the two Aqua CERES instruments in the same way. These results are currently under evaluation 

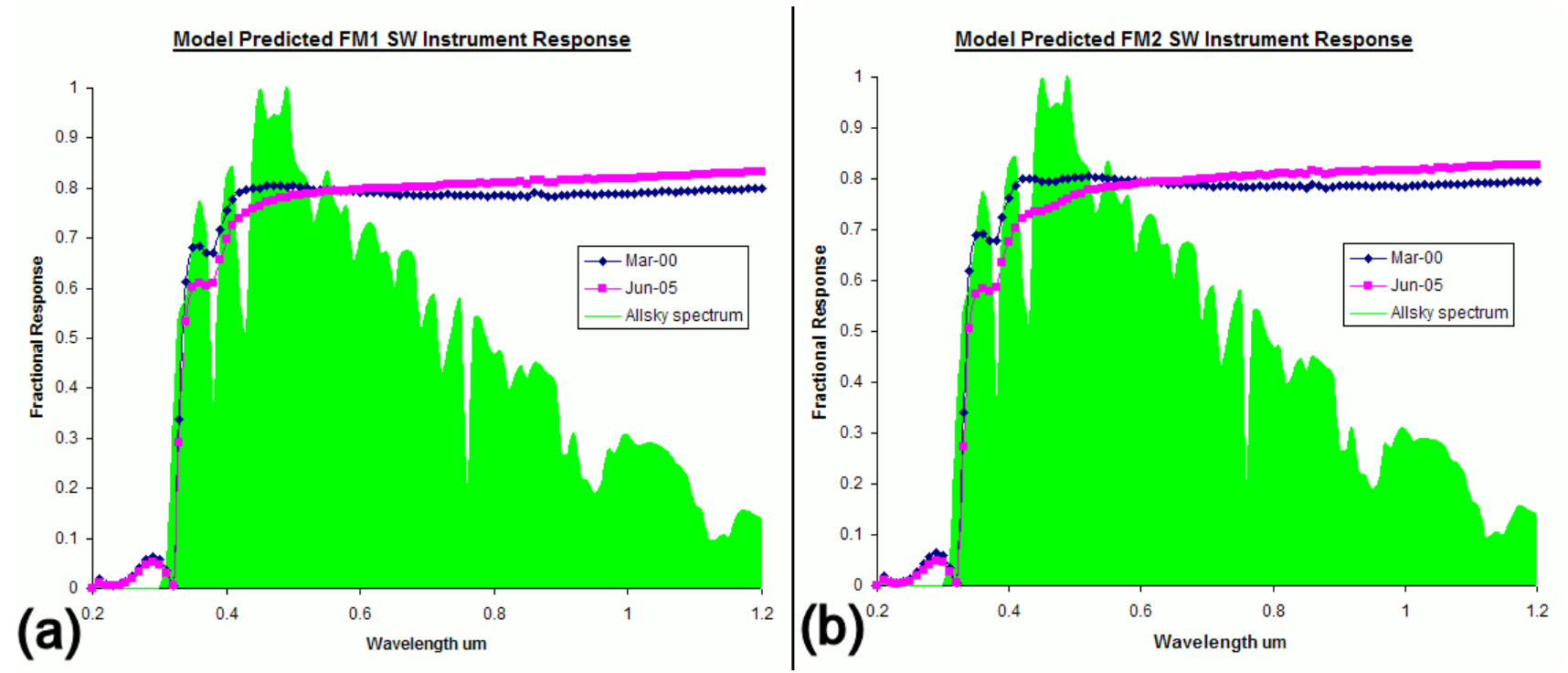

Figure 7. (a) Model derived FM1 SW channel instrument response in June 05 compared to start of mission. (b) Model derived FM2 SW channel instrument response in June 05 compared to start of mission. .

by the CERES instrument working group to determine if they fully compensate for the spectral darkening effects that caused spurious trends in the Edition 2 data release.

\section{SUMMARY}

This study detailed a contamination model developed to assign coloration to the changes occurring in the response of a broad band satellite radiometer subject to spectral darkening. By using co-located imager data, scene types were identified and assigned a spectral signature. The scene type of tropical Deep Convective Clouds was chosen as a stability metric, under the assumption that its albedo remains constant. Hence monitoring the instrument response to reflections from these ice particles on the edge of space serves the purpose originally intended for solar calibrations using the on-board MAM reflectors.

The model then assumes partially polymerized contaminant arrives on the CERES SW filter only when the telescope is exposed to the ram direction. Once fixed to the filter, the contaminant molecules continue to polymerize when exposed to earth scattered UV, decreasing further the instrument response to blue/UV radiance. Given model estimates of contaminant transmission, the assumed constant DCC albedo is used to derive the change in instrument radiometric gain. Tuning of the model matches its estimates of allsky and clear ocean direct compare data between neighboring CERES instruments with the actual measured values. These two direct compare scenes types are chosen because they have radically different spectra, hence using them as a tuning boundary condition ensures accuracy in the spectral shape of the change to the instrument response.

The model derived calibration parameters are currently under evaluation by selected validation studies. The actual maximum possible stability yielded using DCC albedo will also need to be determined by a future study. If the method presented here proves successful and is used to derive CERES calibration, this will be required in order to determine if the stability target of $0.3 \%$ per decade is indeed being met.

\section{ACKNOWLEDGMENTS}

Thanks to Gale Harvey and Carl Maag. This work was supported by the NASA Science Mission Directorate.

\section{REFERENCES}

1. G. Ohring, B. A. Wielicki, R. Spencer, B. Emery, and R. Datla, "Satellite instrument calibration for measuring global climate change," Bulletin of American Meteorological Society 86(9), pp. 1303-1313, 2005. 
2. B. A. Wielicki, B. R. Barkstrom, E. F. Harrison, R. B. Lee, G. L. Smith, and J. E. Cooper, "Clouds and the earth's radiant energy system (ceres): An earth observing experiment," Bulletin of American Meteorological Society 77, pp. 853-868, 1996.

3. G. Matthews, K. Priestley, P. Spence, D. Cooper, and D. Walikainen, "Compensation for spectral darkening of short wave optics occurring on the cloud's and the earth's radiant energy system," in Earth Observing Systems X, J. J. Butler, ed., Proc. SPIE 5882, p. 12, 2005.

4. "Ceres es8 terra or aqua edition2 data quality summary," in http://eosweb.larc.nasa.gov/PRODOCS/ceres/ES8/Quality_Summaries/CER_ES8_Terra_Edition2.html or http://eosweb.larc.nasa.gov/PRODOCS/ceres/ES8/Quality_Summaries/CER_ES8_Aqua_Edition2.html,

5. B. A. Wielicki, T. M. Wong, N. G. Loeb, P. Minnis, K. Priestley, and R. Kandel, "Changes in earth's albedo measured by satellite," Science 308(5723), pp. 825-825, 2005.

6. N. G. Loeb, B. A. Wielicki, W. Su, K. Loukachine, W. Sun, T. M. Wong, K. Priestley, G. Matthews, W. F. Miller, and R. Davies, "Multi-instrument comparison of top-of-atmosphere reflected solar radiation," Journal of Climate inpress, 2006.

7. R. N. Green and P. O. Hinton, "Estimation of angular distribution models from radiance pairs," Journal of Geophysical Research 101 D12, pp. 16951-16959, 1996.

8. P. Spence, K. Priestley, E. Kizer, S. Thomas, D. Cooper, and D. Walikainen, "Correction of drifts in the measurements of the clouds and the earth's radiant energy system scanning thermistor bolometer instruments on the terra and aqua satellites," in Earth Observing Systems IX, W. L. Barnes and J. J. Butler, eds., Proc. SPIE 5542, pp. 53-64, 2004.

9. L. G. Clark and J. D. Dibattista, "Space qualification of optical-instruments using nasa long duration exposure facility," Optical Engineering 17 (5), pp. 547-552, 1978.

10. S. B. Hooker, W. E. Esaias, G. C. Feldman, W. W. Gregg, and C. R. McClain, "An overview of seawifs and ocean color," in SeaWiFS Tech. Rep. Ser., NASA Tech memo Vol 1 104566, 1992.

11. R. A. Barnes, R. E. Eplee, F. S. Patt, H. H. Kieffer, T. C. Stone, G. Meister, J. J. Butler, and C. R. McClain, "Comparison of seawifs measurements of the moon with the u.s. geological survey lunar model," Applied Optics 43(31), pp. 5838-5854, 2004.

12. V. V. Salomonson, W. L. Barnes, P. W. Maymon, H. E. Montgomery, and H. Ostrow, "Modis: Advanced facility instrument for studies of the earth as a system," IEEE Transactions on Geoscience and Remote Sensing 27(2), pp. 145-153, 1989.

13. N. G. Loeb, K. J. Priestley, D. P. Kratz, E. B. Geier, R. N. Green, and B. A. Wielicki, "Determination of unfiltered radiances from the clouds and the earth's radiant energy system instrument," Journal of Applied Meteorology 40, pp. 822-835, 2001.

14. C. F. Ohlich, "Observations of irradiance variations," in Space Science Rev., 94, pp. 15-24, 2000.

15. A. Hahne, A. Lefebvre, and J. Callies, "Gome - a new instrument for ers-2," ESA Bulletin-European Space Agency 73, pp. 22-29, 1993.

16. G. Matthews, K. Priestley, and P. Spence, "Z-domain numerical filter for removal of thermistor bolometer slow mode transients," in Earth Observing Systems X, J. J. Butler, ed., Proc. SPIE 5882, p. 13, 2005. 